\title{
MIOCARDIOPATIA NÃO COMPACTADA: UM RELATO DE CASO
}

\section{NON-COMPACTION CARDIOMYOPATHY: A CASE REPORT}

\author{
Carla Scuderi1*, Ana Paula Susin Osório², Leandro Shigueru Ikuta Ueda ${ }^{3}$
}

\begin{abstract}
${ }^{1}$ Acadêmica de Medicina da Faculdade Assis Gurgacz. ${ }^{2}$ Docente do curso de Medicina do Centro Universitário Assis Gurgacz, mestre em Ciências da Saúde (Cardiologia pelo Instituto de Cardiologia do Rio Grande do Sul) e cardiologista do Corpo Clínico do Hospital São Lucas. ${ }^{3}$ Docente do curso de Medicina do Centro universitário Assis Gurgacz e cardiologista do Corpo Clínico do Hospital São Lucas.
\end{abstract}

*Autor correspondente: carlascuderii@hotmail.com , https://orcid.org/0000-0002-9677-9893

\section{RESUMO}

Miocárdio não-compactado (MNC) é uma cardiopatia congênita com incidência rara, de difícil diagnóstico, sendo assim, seu tratamento e prognóstico ainda não são tão disseminados. Por ser uma alteração genética pode acometer todas os gêneros, porém, é mais prevalente em homens. Tem como quadro clínico uma apresentação variável, desde de caráter assintomático, como também insuficiência cardíaca congestiva, arritmias e tromboembolismo sistêmico. Os achados ecocardiográficos e de ressonância magnética cardíaca permitem um diagnóstico precoce e tratamento adequado. O melhor conhecimento acerca da doença, o estabelecimento de critérios diagnósticos e forma de manejo têm sido decisivos para o diagnóstico precoce e prognóstico favorável. No caso apresentado, é relatado um paciente adulto masculino portador de MNC diagnosticado aos 45 anos após exames de rotina, já que este era assintomático. Seguimento deu-se através de exame de imagem com ecocardiograma transtorácico anualmente.

Palavras-chave: Cardiopatia congênita, miocardiopatia não compactada, diagnóstico

\begin{abstract}
Non-compaction cardiomyopathy (NCCM) is a rare congenital heart disease that is difficult to diagnose, and its treatment and prognosis are not as widespread. Because it is a genetic alteration, it can affect all genders, but it is more prevalent in men. Its clinical presentation has a variable presentation, from asymptomatic character, as well as congestive heart failure, arrhythmias and systemic thromboembolism. Echocardiographic and cardiac magnetic resonance imaging findings allow early diagnosis and appropriate treatment. Better knowledge about the disease, the establishment of diagnostic criteria and management methods have been decisive for early diagnosis and favorable prognosis. In the case presented here, we report a male adult patient with NCCM diagnosed at 45 years after routine exams, as he was asymptomatic. Follow-up was performed by transthoracic echocardiography.
\end{abstract}

Keywords: Congenic cardiopatics, Non-compaction cardiomyipathy, diagnosis.

\section{INTRODUÇÃO}

A miocardiopatia não compactada ( $\mathrm{MNC}$ ) é uma doença rara que ocorre, provavelmente, durante a compactação do miocárdio no início do desenvolvimento fetal, durante o período embrionário. tem por definição ser isolada, já que, é comum o 
diagnóstico em pacientes adultos que não apresentam outra doença cardíaca (ROSA, L.V. et al., 2011). As manifestações podem ser desde assintomáticas a sintomáticas, sendo elas: insuficiência cardíaca congestiva, arritmias e tromboembolismo sistêmico (ROSA, L.V. et al., 2011).

O diagnóstico da MNC é realizado frequentemente pela ecocardiografia. Outros métodos de imagem como a ressonância magnética (RNM), sendo o método mais escolhido, tomografia computadorizada e ventriculografia esquerda fazem 0 diagnóstico ou confirmam a suspeita clínica (OECHSLIN, E.N. et al., 2000)

O caso reportado é de um adulto jovem, assintomático, que foi diagnosticado em consulta de rotina. O diagnóstico se torna primordial, a partir do momento que é constatado que parte das MNC podem evoluir para insuficiência cardíaca, bem como o tromboembolismo pode ocasionar AVC isquêmico, isquemia mesentérica, embolia periférica de membros superiores e inferiores, da mesma forma que as arritmias têm importância clinica já que estas podem provocar parada cardíaca e morte súbita. Estas patologias são graves e necessitam de intervenção precoce. Sendo assim, o reconhecimento a cerca dessa patologia é relevante tanto para retardar a evolução de uma possível insuficiência cardíaca quanto para evitar as arritmias e o tromboembolismo e, também, para melhor prognóstico da doença.

Dessa forma, a abordagem do assunto se torna valoroso, visto que, com a melhora dos métodos de imagem há, também, um maior número de diagnósticos ressaltando a necessidade de ter um conhecimento cada vez mais amplo e mais especifico sobre a patologia, afim de poder instituir o tratamento de maneira correta.

\section{METODOLOGIA}

O relato trata-se de uma pesquisa de abordagem qualitativa, realizada por meio da análise documental da evolução do quadro clínico de um paciente adulto jovem, do gênero masculino, atendido na Pro Cardíaco Cascavel, localizado na cidade de Cascavel-PR, portador de Miocardiopatia Não Compactada. Tendo em vista sua raridade, o relato de caso tem valor crucial para ampliar os conhecimentos referentes à manifestação, evolução e opções terapêuticas da patologia em estudo, para o reconhecimento rápido, garantindo um diagnóstico mais preciso e precocemente possível, com finalidade de melhorar o prognóstico e ampliar as opções de abordagem terapêutica.

Os dados utilizados neste trabalho foram obtidos através de análise documental, a qual se baseia no estudo detalhado do prontuário do paciente, além de análise dos exames laboratoriais e de imagem, a fim de obter informações precisas sobre as queixas clínicas, tratamento e conduta médica a que o paciente tenha sido submetido, visto tratar-se de pesquisa com ser humano, tendo como preocupação a preservação da identidade do paciente.

Ressalta-se que o projeto de pesquisa foi aprovado pelo Comitê de Ética em Pesquisa em Seres Humanos da Faculdade Assis Gurgacz- FAG por meio do Parecer № 08393618.1.0000.5219, estando em cumprimento com a Resolução 196/96 do Conselho Nacional de Saúde.

\section{REFERENCIAL TEÓRICO}

A miocardiopatia não compactada (MNC) é uma desordem rara, que ocorre provavelmente durante a compactação do miocárdio no início do desenvolvimento 
fetal, durante o período embrionário. Essa miocardiopatia contém trabeculações miocárdicas proeminente com recessos intrabeculares profundos e espessamento do miocárdio em duas camadas diferentes: a compactada e a não compactada. Tem por definição ser isolada, já que, é comum o diagnóstico em pacientes adultos que não apresentam outra doença cardíaca. As manifestações podem ser desde assintomáticas a sintomáticas, sendo elas: insuficiência cardíaca congestiva, arritmias e tromboembolismo sistêmico (ROSA, L. V. et al., 2011). Ainda, foi encontrada uma relação entre MNC e disfunções neuromusculares, sendo que em torno de $82 \%$ dos pacientes apresentam algum tipo de manifestação neuromuscular (STOLLBERGER, C.; FINSTERER, J.; BLAZEK, G, 2002)

Tem-se como características a alteração na parede miocárdica devido à proeminência de suas trabeculações com recessos intertrabeculares profundos que podem ser secundários à parada intrauterina da compactação miocárdica que ocorre nos primórdios do desenvolvimento fetal; o resultado são duas lâminas apresentadas pelo miocárdio, em que uma é compactada, e a outra, não compactada (WEIFORD, B.C. et al., 2004)

Sexo masculino é o mais afetado, sendo que representam $56 \%$ a $82 \%$ dos casos no quadro de MNC isolada (CHIN, T.K. et al., 1990), sendo possível ocorrer episódio familiar entre 18 a $50 \%$ dos casos (ARBUSTINI, E. et al., 2016).

O diagnóstico da MNC é realizado, geralmente, pela ecocardiografia. Outros métodos de imagem como a ressonância magnética (RNM), sendo o método mais escolhido, tomografia computadorizada e ventriculografia esquerda fazem 0 diagnóstico ou confirmam a suspeita clínica. A ecocardiografia é utilizada como método diagnóstico e de acompanhamento (OECHSLIN, E.N. et at., 2000).

Segundo, JENNI, R. et al., 2001, um dos critérios mais utilizados, tem como analise: 1) existência de duas camadas, uma das quais fina e compacta (C) e a outra não compactada (NC), que, quando medidas em telessístole no paraesternal curto eixo, apresentam uma razão $\mathrm{NC} / \mathrm{C}>2 ; 2$ ) ausência de anomalias estruturais cardíacas coexistentes; 3) numerosas trabeculações excessivamente proeminentes e recessos intertrabeculares profundos; 4) recessos perfundidos por sangue, com fluxo demonstrável no Doppler. Ainda, sendo necessário a exclusão de outra cardiopatia pela própria patogenia da MNC. Uma relação do miocárdio não compactado ao compactado $>2,3$ produz maior sensibilidade (86\%) e especificidade (99\%) no diagnóstico $^{10}$ (8) (ARBUSTINI, E. et al., 2016). Geralmente, a relação do miocárdio não compactado para o compactado sendo superior a dois é diagnóstico de MNC pela ecocardiografia. No entanto, a ecocardiografia, muitas vezes, pode não analisar a região apical de melhor maneira, levando a um diagnóstico errôneo do grau de compactação ventricular esquerda. Dessa forma, a RNM é considerada o método de escolha para confirmar ou afastar o diagnóstico da MNC, pois oferece de forma detalhada a morfologia cardíaca (JENNI, R. et al, 2001.)

O tratamento é sintomático, relacionado as principais manifestações da doença sendo elas o tromboembolismo, arritmias e insuficiência cardíaca progressiva (ROSA, L. V. et al., 2011). Dessa forma, diversos autores defendem o uso de anticoagulação oral profilática em todos os doentes, tendo a presença ou não de trombos (RITTER, M. et al., 1997). Atualmente, o risco de tromboembolismo é menor do que se cogitava anteriormente, com isso alguns diretrizes recomendam anticoagulação aos pacientes que apresentam função sistólica diminuída com fração de ejeção inferior a 40\%, história de tromboembolismo ou fibrilação atrial (MURPHY, R.T. et al.,2005). Pacientes que no momento do diagnóstico apresentam função sistólica preservada e estão assintomáticos têm um prognóstico mais favorável (ZHANG, X., 2016). 


\section{ANÁLISES E DISCUSSÃO DOS RESULTADOS}

\subsection{RELATO DE CASO}

G. M. S., masculino, 45 anos, atleta de corrida com treinos regulares de 10-20 $\mathrm{km}$, em consulta de rotina, assintomático, sem doenças prévias, não faz uso de medicamentos, nega dor precordial e dispneia aos esforços, nega tabagismo e etilismo, sem histórico familiar de cardiopatia e com exame físico sem alterações. Realizado exames de rotina com aumento de LDL, Eletrocardiograma (ECG) com resultado sinusal normal, Teste de Esforço Cardiopulmonar (TCP) com boa aptidão e ausência de arritmias, Ecocardiograma (ECO) com hipertrofia do VE com hipertrabeculação de predomínio apical sendo a função sistólica global preservada, sugerindo miocardiopatia não compactada (MNC).

Solicitado Ressonância Magnética Cardíaca (RNM) para confirmação diagnostica com ventrículo esquerdo (VE) com diâmetros aumentados e espessura normal, aumento da trabeculação adjacente às paredes anterior, lateral e médio apical (imagem 1,2 e 3) com maior relação de miocardio não compactado em 5 na parede anterior medial sendo o normal $<2,3$, alteração de relaxamento do VE, aorta e pericárdio normais, confirmando o resultado de MNC. Orientado retorno em 60 dias para repetir TCP e anualmente ECO. Paciente segue sem alterações em exame físico e assintomático do ponto de vista cardiovascular.

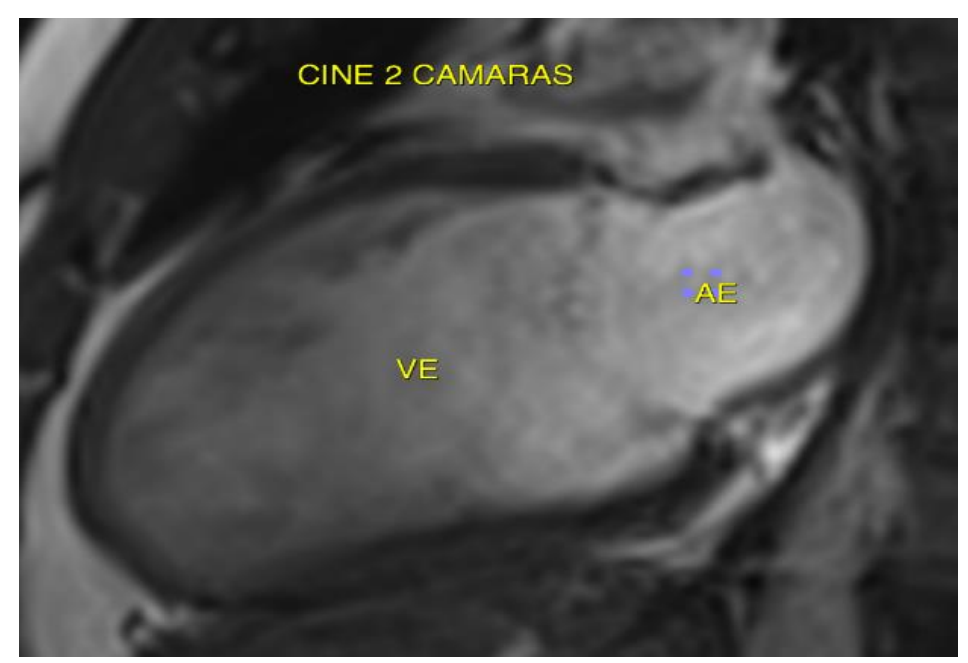

Figura 1. Imagem de ressonância magnética cardíaca em cine 2 câmaras mostrando as câmaras cardíacas esquerdas e a hipertrabeculação em porções medio-apical em ventrículo esquerdo 


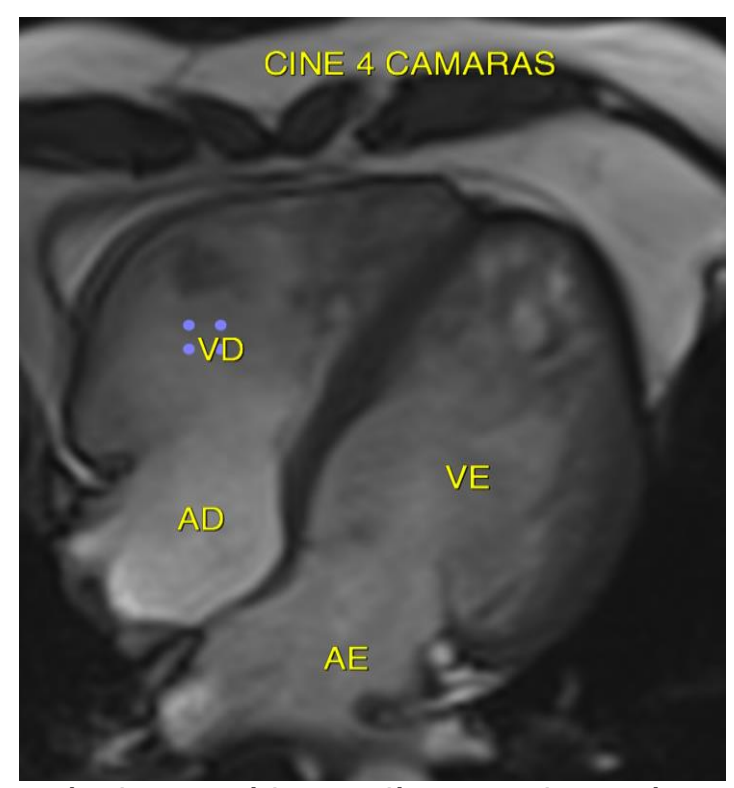

Figura 2. Imagem de ressonância magnética cardíaca em cine 4 câmaras mostrando as câmaras cardíacas atriais e ventriculares e a hipertrabeculação em porções medio-apical em ventrículo esquerdo.

\subsection{DISCUSSÃO}

O sexo masculino é o mais afetado, sendo que representam $56 \%$ a $82 \%$ dos casos no quadro de MNC isolada (CHIN, T.K. et al., 1990), sendo possível ocorrer episódio familiar entre 18 a 50\% dos casos (ARBUSTINI, E. et al., 2016).

O paciente em questão se encontra assintomático, sem doença previa, sem antecedente familiar de cardiopatia preenchendo apenas o critério de gênero para a doença. Suspeitou-se da doença quando exames de rotina apontaram MNC pelo Ecocardiograma, a qual foi confirmada pela RNM ao mostrar a relação do miocárdio compactado pelo não compactado em 5, sendo que, como dito, uma relação do miocárdio não compactado ao compactado $>2,3$ produz maior sensibilidade $(86 \%)$ e especificidade (99\%) no diagnóstico (ARBUSTINI, E. et al., 2016).

Por ser assintomático, o seguimento deu-se apenas com ECO anualmente, e pela função sistólica preservada foi optado em não dar medicação. Pelos achados de exame e pela sintomatologia clínica inexistente, paciente tem um bom prognóstico, já que pacientes que no momento do diagnóstico apresentam função sistólica preservada e estão assintomáticos têm um prognóstico mais favorável (ZHANG, X., 2016).

\section{CONSIDERAÇÕES FINAIS}

A MNC apresenta difícil diagnóstico, sendo importante o conhecimento acerca da patologia para ser possível o diagnóstico precoce e tratamento nos casos necessários. Os achados do ecocardiograma e ressonância magnética cardíaca foram evidentes para a MNC, os quais condizem com todos os critérios para o diagnóstico.

Como demonstrado nesse caso, o paciente ser assintomático requer muito mais agilidade na investigação, já que ele não possui nenhuma sintomatologia que possa indicar a MNC. Mesmo nesses casos, a associação entre ecocardiograma e ressonância nuclear magnética são suficientes para confirmar a doença. Sendo assim, 
o tratamento e seguimento se deu com acompanhamento e ecocardiograma transtorácico anualmente.

\section{REFERÊNCIAS}

ARBUSTINI, E. et al. Left ventricular noncompaction: a distinct genetic cardiomyopathy?. J Am Coll Cardiol, vol 68, n. 9, p. 949-66. Aug, 2016 https://doi.org/10.1016/.j.jacc.2016.05.096

CHIN, T.K. et al. Perloff JK, Williams RG, Jue J, Mohrmann R. Isolated noncompaction of left ventricular myocardium: a study of eight cases. Circulation, vol. 82, n. 2, p. 50713. Aug, 1990

https://doi.org/10.1161/01.cir.82.2.507

JENNI, R. et al. Echocardiographic and pathoanatomical characteristics of isolated left ventricular non-compaction: a step towards classification as a distinct cardiomyopathy. Heart, vol 86, n. 6, p. 666-71. Dec, 2001

https://doi.org/10.1136/heart.86.6.666

MURPHY, R.T. et al. Natural history and familial characteristics of isolated left ventricular non-compaction. Eur Heart J, vol 26, n. 2, p. 187-92. Jan, 2005 https://doi.org/10.1093/eurheartj/ehi025

OECHSLIN, E.N. et al. Long-term follow-up of 34 adults with isolated left ventricular noncompaction: a distinct cardiomyopathy with poor prognosis. J Am Coll Cardiol, vol. 36, n. 2, p. 493-500. Aug, 2000

https://doi.org/10.1016/S0735-1097(00)00755-5

RITTER, M. et al. Isolated noncompaction of the myocardium in adults. Mayo Clin Proc, vol. 72, n. 1, p. 26-31. Jan, 1997

https://doi.org/10.4065/72.1.26

ROSA, L. V. et al. Miocardiopatia não compactada: uma visão atual. Arq. Bras. Cardiol. v. 97, n. 1, p. e13-e19. July, 2011

http://dx.doi.org/10.1590/S0066-782X2011000900021

STOLLBERGER, C.; FINSTERER, J.; BLAZEK, G.; Left ventricular hypertrabeculation/noncompaction and association with addi tional cardiac abnormali ties and neuromuscular disorders. Am J Cardiol, vol 90, n. 8, p. 899-902. Oct, 2002 https://doi.org/10.1016/s0002-9149(02)02723-6

WEIFORD, B.C.; SUBBARAO, V.D.; MULHERN, K.M.; Noncompaction of the ventricular myocardium. Circulation, vol. 109, n. 24, p. 2965-71. Jun, 2004 https://doi.org/10.1161/01.CIR.0000132478.60674.D0

ZHANG, X. et al. Incremental value of contrast echocardiography in the diagnosis of left ventricular noncompaction. Front Med, vol 10, n. 4, p. 499-506. Dec, 2016 https://doi.org/10.1007/s11684-016-0473-8 